\title{
Uptake of gases in bundles of carbon nanotubes
}

\author{
George $\operatorname{Stan}^{1,3,6}$, Mary J. Bojan ${ }^{2}$, Stefano Curtarolo ${ }^{1,4}$, \\ Silvina M. Gatica ${ }^{1,5}$, and Milton W. Cole ${ }^{1}$ \\ ${ }^{1}$ Department of Physics, Penn State University, University Park, PA 16802, USA \\ ${ }^{2}$ Department of Chemistry, Penn State University, University Park, PA 16802, USA \\ ${ }^{3}$ Present address: Institute for Physical Science \& Technology and \\ Department of Chemical Engineering, University of Maryland, College Park, MD 20742, USA \\ ${ }^{4}$ Present address: Department of Materials Science and Engineering, \\ MIT, Cambridge, MA 02139, USA \\ ${ }^{5}$ Permananent address: Departamento de Física, Facultad de Ciencias Exactas y Naturales, \\ Universidad de Buenos Aires, Buenos Aires, Argentina. \\ ${ }^{6}$ corresponding author, e-mail: gns@ipst.umd.edu, phone: (301) 405-4812, fax: (301) 314-9404
}

(October 29, 2018)

\begin{abstract}
Model calculations are presented which predict whether or not an arbitrary gas experiences significant absorption within carbon nanotubes and/or bundles of nanotubes. The potentials used in these calculations assume a conventional form, based on a sum of two-body interactions with individual carbon atoms; the latter employ energy and distance parameters which are derived from empirical combining rules. The results confirm intuitive expectation that small atoms and molecules are absorbed within both the interstitial channels and the tubes, while large atoms and molecules are absorbed almost exclusively within the tubes.
\end{abstract}

\section{INTRODUCTION}

The absorption of gases in nanopores is a subject of growing experimental and theoretical interest, stimulated by both fundamental scientific questions and the potential for many technologies [1]. One of the most important questions to be addressed is whether or 
not a specific gas is significantly absorbed within carbon nanotubes; we will define the word "significant" in Eq. 2 below. While the answer depends in detail on the specific thermodynamic conditions of the coexisting vapor (pressure $P$ and temperature $T$ ), one expects that intuitive considerations based on size and energy scales ought to provide useful qualitative insights. For example, it has been demonstrated that gases whose condensed phases possess low surface tensions are strongly imbibed in these tubes [22]. This important result can be understood from either the Kelvin equation or a comparison of competing interaction (adhesive vs. cohesive) energies. These considerations arise in the analogous problem of wetting transitions [23].

This paper addresses this basic question by employing a simple, but plausible, model of the interaction potential, from which we compute the adsorption as a function of $P$ and $T$. We assume that the adsorption potential can be derived from a sum of Lennard-Jones (LJ) two-body interactions between the host $\mathrm{C}$ atoms and the adsorbate. This pair potential has distance and energy parameters obtained with semiempirical combining rules from the LJ $\epsilon$ and $\sigma$ parameters of the $\mathrm{C}$ atoms and the adsorbate [24 27]:

$$
\begin{aligned}
\sigma_{g C} & =\frac{\sigma_{g g}+\sigma_{C C}}{2} \\
\epsilon_{g C} & =\sqrt{\epsilon_{g g} \epsilon_{C C}}
\end{aligned}
$$

where "g" and "C" refer to the gas and C atoms, respectively. Estimates of the gas parameters are given for some relevant systems in Table [ [28,29,44, while for C atoms we use $\sigma_{C C}=3.4 \AA$ and $\epsilon_{C C}=28 \mathrm{~K}$ [25]. These values are typical, but uncertain within a $15 \%$ range [30].

This paper's approach is the following. We first choose a particular (somewhat arbitrary) criterion for calling the uptake "significant". For example, Fig. 1(a) and most of our work employ the criterion

$$
\rho^{*}=\rho \sigma_{g C}=0.1
$$

where $\rho=N / L$ is the one-dimensional (1d) density, with $N$ the number of adsorbed atoms and $L$ the length of the tube, and $\rho^{*}$ is the corresponding dimensionless density. For gases of interest here, this criterion corresponds to a mean $1 \mathrm{~d}$ spacing of order $30 \AA$. This is a 
very low density. Although we do consider more stringent criteria elsewhere in this paper, the results do not differ qualitatively. The reason for the lack of sensitivity to the threshold is that once adsorption commences, it rises rapidly as a function of $P$ (until the crowding effect of repulsive forces slows the variation of coverage with $P$ ).

In the nanotube bundle geometry, adsorption can take place inside the tubes, in the interstitial channels, and on the outer surface of the nanotube bundle (Fig. 2). Typical length scales for the triangular lattice of nanotubes in the bundle are: lattice constant $17 \AA$, nanotube radius $6.9 \AA$, bundle diameter between 50 and $100 \AA$, and bundle length $\sim 10-100 \mu \mathrm{m}$ [36]. We will see that size is a critical variable determining uptake. Some key findings of this paper appear in Fig. 1(a), which shows the uptake at a very small ratio of $P$ to saturated vapor pressure $\left(P_{0}\right)$. Small atoms and molecules (which typically have small values of $\left.\epsilon_{g g}\right)$ are strongly adsorbed within both the nanotubes and the narrow interstitial channels (IC's) between nanotubes. Larger particles, in contrast, do not "fit" within the IC's but do imbibe within the tubes. Perhaps a surprising feature of Fig. 11(a) is that a hypothetical gas with a very large value of $\epsilon_{g g}$ adsorbs in neither place. This occurs because the relative tendency (compared with bulk condensation) of a gas to be absorbed within the tube at a given undersaturation depends on the ratio of adhesive to cohesive energies. The geometric mean combining rule for $\epsilon_{g C}$ implies that this ratio varies as the inverse square root of $\epsilon_{g g}$, so a large $\epsilon_{g g}$ implies small uptake. This finding is qualitatively consistent with the empirical correlation between uptake and surface tension mentioned above. It also correlates with the physics determining wetting behavior of liquids for which the analogous comparison involves the same kind of interaction ratio [23].

This paper makes a number of simplifications in order to draw such general conclusions. Arguably the most drastic assumptions are that the nanotubes are infinite and perfect and that the nanotube bundles involve a unique species of tubes in a regular array (geometry unaffected by the adsorption).

The outline of this paper is the following. Section II describes our model of the interactions. Section [II presents the statistical mechanical model used in the calculations. Section IV reports our results. Section $\square$ summarizes these and discusses open questions. 


\section{ADSORPTION POTENTIAL}

A basic assumption in our model is that the potential energy experienced by a molecule at position $\mathbf{r}$ can be evaluated by a summation of two-body interactions $U(\mathbf{x})$ between the molecule and the carbon atoms comprising the tube:

$$
V(\mathbf{r})=\sum_{i} U\left(\mathbf{r}-\mathbf{R}_{\mathbf{i}}\right)
$$

This assumption is made in the overwhelming majority of calculations of gas interactions with either graphite and carbon nanotubes. In the graphite case, many-body effects have been found to be $\sim 15 \%$ corrections to ab initio pair potential sums [32]. Hence, the empirical pair potential should be regarded as an effective pair potential. One might expect somewhat smaller many body contributions in the nanotube case because the molecule is somewhat farther from the nearest carbon atom [33] and because the effective coordination number is larger in the nanotube case than on graphite. In contrast, the argument in the IC case leads to the prediction of a larger many body effect than on graphite. These expectations, however, might not be correct because the many-body expansion involves geometry-dependent competing terms of opposite signs [34 and because the two body energy for the IC is typically of much larger magnitude than on a flat surface.

Another key assumption made here is that the pair potential is isotropic and of LJ form: $U(x)=4 \epsilon\left[(\sigma / x)^{12}-(\sigma / x)^{6}\right]$. There is ab initio and empirical evidence to the effect that anisotropy of the pair potential plays a role in adsorption potentials on graphite [35]. Nevertheless, most studies of adsorption on that surface neglect such an effect and use a LJ pair potential similar to what we use here. The final assumption is the use of an azimuthally and longitudinally averaged potential. The potential at distance $r$ from the axis of the cylinder is then [12]:

$$
V(r ; R)=3 \pi \theta \in \sigma^{2}\left[\frac{21}{32}\left(\frac{\sigma}{R}\right)^{10} f_{11}(x) M_{11}(x)-\left(\frac{\sigma}{R}\right)^{4} f_{5}(x) M_{5}(x)\right]
$$

where $\theta=0.38 \AA^{-2}$ is the surface density of $\mathrm{C}$ atoms and $R$ is the radius of the cylinder. Here, $x=r_{<} / r_{>}$, and $r_{<(>)}$are the smaller (greater) of $r$ and $R$. The function $f_{n}(x)$ is

defined as 1 for $r<R$ and $(R / r)^{n}$ for $r>R$, with $n$ a positive integer. Here we use the integrals 


$$
M_{n}(x)=\int_{0}^{\pi} d \varphi \frac{1}{\left(1+x^{2}-2 x \cos \varphi\right)^{n / 2}}
$$

We emphasize that each approximation introduces an error, but the qualitative trends ought to be reliable. At this time, the lack of high quality ab initio calculations would seem to warrant this kind of approach.

The IC potential is obtained by summing up the contribution from three nanotubes and azimuthally averaging the result. Figures 35 show contour plots in the $\sigma_{g g}-\epsilon_{g g}$ plane of the reduced minimum of the adsorption potential $\left(V_{\min }^{*} \equiv V_{\min } / \epsilon_{g g}\right)$ for all of these sites. Inside both the tubes and in IC's there is a threshold $\sigma_{g g}$ value above which the potential becomes repulsive, corresponding to gases which are too big to fit in these restricted geometries; these thresholds are $\sigma_{g g} \simeq 11.4 \AA$ (tubes) and $3.4 \AA$ (IC's) for nanotubes of radius $6.9 \AA$ studied here. Outside of the bundle, there are no such size constraints for the adsorbed atoms/molecules, as the adsorbate can always find a region in which the potential is attractive; at a fixed value of $\epsilon_{g g}$ large systems yield larger $\left|V_{m i n}^{*}\right|$ due to their larger coordination number of $\mathrm{C}$ atoms. In all three cases the most negative values of $V_{\min }^{*}$ occur for small values of $\epsilon_{g g}$. In the tube and external surface cases, but not the IC case, the most negative values of $V_{\min }^{*}$ occur for large $\sigma_{g g}(\gtrsim 9 \AA)$.

\section{STATISTICAL MECHANICS}

Our interest is whether atoms are likely to go inside the tubes, in the interstitial channels, and on the outer surface of the nanotube bundle. This behavior is determined by the thermodynamic conditions $(P, T)$ and microscopic parameters (especially $\sigma_{g g}$ relative to $R$ ). A key factor implicit here is the cohesive energy of the bulk phase of the adsorbate which determines a relevant pressure, i. e. saturated vapor pressure $P_{0}$. We construct a simple model for the low coverage regime of atoms inside nanotubes, neglecting the interactions between adsorbate atoms, while for atoms moving in the very confining IC's any density can be considered because of the mathematical simplicity resulting from the $1 \mathrm{~d}$ character of the system. We have discussed elsewhere the extreme quantum behavior of He at low T [14, 37. In the present case we assume that classical statistical mechanics applies [38]. 
We now compute the chemical potential $\mu$ of the adsorbate. All of our calculations take the coexisting three-dimensional vapor to be an ideal gas, so that the chemical potential can be expressed in terms of pressure as $\mu=\beta^{-1} \ln \left(\beta P \lambda^{3}\right)$. Here $\beta^{-1}=k_{B} T$, and $\lambda=$ $\left(2 \pi \hbar^{2} \beta / m\right)^{1 / 2}$ is the de Broglie thermal wavelength for particles of mass $m$. It is convenient to measure the chemical potential with respect to its value at saturation, $\mu_{0}$,

$$
\Delta \mu=\mu-\mu_{0}=\beta^{-1} \ln \left(P / P_{0}\right)
$$

An analytical expression for $P_{0}$ is available from computer simulation data of the LennardJones system's liquid-vapor coexistence [39], $\ln P_{0}^{*}=1.2629 T^{*}-4.9095 / T^{*}-0.15115 / T^{* 4}$, where $P_{0}^{*}=P_{0} \sigma_{g g}^{3} / \epsilon_{g g}$ and $T^{*}=k_{B} T / \epsilon_{g g}$ are reduced quantities.

Consider first the adsorption inside a single nanotube. The chemical potential of the ideal gas in an external potential can be expressed as a function of the number of adsorbed atoms, $N$, and temperature:

$$
e^{\beta \mu}=\frac{N \lambda^{3}}{\int_{N T} d \mathbf{r} \exp (-\beta V(r))}
$$

where the integral is performed over the volume of the nanotube. This is an application of Henry's law. Then, the chemical potential relative to its value at saturation assumes a simple form due to the cylindrical symmetry of the adsorption potential:

$$
\Delta \mu=\beta^{-1} \ln \left(\frac{\rho}{2 \pi \beta P_{0} \int_{N T} d r r \exp (-\beta V(r))}\right)
$$

Atoms in the narrow IC's are strongly confined to the vicinity of the axis so that a 1d model is applicable and solvable for all densities. As previously discussed in the case of very small nanotubes [12], the transverse motion may be treated independently of the longitudinal motion and the chemical potential in this case has the form:

$$
\mu=\mu_{\perp}+\mu_{1 d}
$$

where $\mu_{\perp}$ is the transverse contribution and $\mu_{1 d}$ is the chemical potential of a $1 \mathrm{~d}$ gas. In general, $\beta \mu_{\perp}=\ln \left(\sum_{i} \exp \left(-\beta \epsilon_{i}\right)\right)$, where $\left\{\epsilon_{i}\right\}_{i=0,1, \ldots}$ is the transverse spectrum of individual atoms/molecules. At low $\mathrm{T}\left(\beta\left(\epsilon_{1}-\epsilon_{0}\right)<<1\right)$, the ground state dominates the sum and $\mu_{\perp} \simeq \epsilon_{0}$. The ground state energy can be determined very accurately using the WKB method 
[40], since the adsorption potential is well-approximated by a parabola in the vicinity of the IC axis [14]. Results for the potential well depths of various gases are shown in Table [.

The 1d chemical potential is obtained by integrating the 1d Gibbs-Duhem relation

$$
\frac{\partial \mu_{1 d}}{\partial P_{1 d}}=\frac{1}{\rho}
$$

where $P_{1 d}$ is the $1 \mathrm{~d}$ pressure. The particle density in the case of only nearest-neighbor interactions is given by the equation of state 41,42

$$
\rho=\frac{\int_{0}^{\infty} d z \exp \left(-\beta\left[u(z)+z P_{1 d}\right]\right)}{\int_{0}^{\infty} d z z \exp \left(-\beta\left[u(z)+z P_{1 d}\right]\right)}
$$

Here $\mathrm{u}(\mathrm{z})$ is the LJ potential describing the interactions between adsorbed atoms. The integration of eq. 10 leads to

$$
\beta \mu_{1 d}=\ln \left(\frac{\beta \lambda P_{1 d, 0} \int_{0}^{\infty} d z \exp \left(-\beta\left[u(z)+z P_{1 d, 0}\right]\right)}{\int_{0}^{\infty} d z \exp \left(-\beta\left[u(z)+z P_{1 d}\right]\right)}\right)
$$

$P_{1 d, 0}$ is an initial low pressure chosen such that the ideal gas limit is reproduced. The density dependence of the $1 \mathrm{~d}$ chemical potential is finally obtained by eliminating the $1 \mathrm{~d}$ pressure between eqs. 10, 11 and 12 .

As shown in Fig. 5, the external surface of the nanotube bundle also provides an attractive domain of adsorption. We have studied adsorption in the very attractive groove-like channel which runs parallel to the nanotube axes, as shown in Figure 2 [13]. Then, a procedure similar to that employed in the case of IC's is applicable to computing the coverage. The contribution of the longitudinal motion to the chemical potential is determined in the same fashion as in the case of IC's. The ground state energy of the transverse motion can be estimated through a parabolic approximation for the adsorption potential at this site. Values of the ground state energy $\left(E_{0}^{e x t}\right)$ obtained in this fashion for the systems studied, as well as the well-depth of the adsorption potential $\left(V_{m i n}^{e x t}\right)$, are listed in Table $\mathbb{q}$.

\section{RESULTS}

The lines obtained by setting the coverage equal to the threshold criterion can be seen in Figures 1(a)-(d). Figure1(a) shows this behavior in the case of $\rho^{*}=0.1$, for $\Delta \mu^{*}=\Delta \mu / \epsilon_{g g}=$ 
-10 and $T^{*}=1$. As expected, small atoms or molecules $\left(\mathrm{He}, \mathrm{Ne}, \mathrm{H}_{2}\right)$ fit easily inside both the tubes and IC's, while large molecules do not fit in the narrow IC's. Hypothetical (but nonexistent) atoms with $\sigma_{g g}<2.5 \AA$ are adsorbed in the IC's only if their self-interaction energy $\left(\epsilon_{g g}\right)$ does not exceed a threshold value. The upper limit to the molecular size for adsorption inside the tubes can be seen in Fig. 6. Indeed, the experimental observation of $\mathrm{C}_{60}$ molecules encapsulated in nanotubes [45] is consistent with this expectation (as the point near $\sigma_{g g} \sim 9 \AA$ indicates).

Including the effect of interactions does not affect our results significantly. In the framework of the gas-surface virial expansion [46],

$$
N \simeq e^{\beta \mu} Q_{1}[1+\rho \eta(T)]
$$

where $Q_{1}$ is the single particle canonical partition function, and

$$
\eta(T)=L \frac{\int_{N T} d \mathbf{r}_{\mathbf{1}} d \mathbf{r}_{\mathbf{2}} \exp \left(-\beta\left(V\left(r_{1}\right)+V\left(r_{2}\right)\right)\left[e^{-\beta u\left(\left|\mathbf{r}_{1}-\mathbf{r}_{\mathbf{2}}\right|\right)}-1\right]\right.}{\left[\int_{N T} d \mathbf{r} \exp (-\beta V(r))\right]^{2}}
$$

The net effect of the virial correction is at most a $0.1 \%$ change of $\Delta \mu$; such a small magnitude is consistent with the expected behavior in the low pressure regime of interest here.

The evolution of the diagram as a function of the adsorption criterion can be seen in Fig. 11(b). As the threshold density decreases $\left(\rho^{*}=0.05\right.$ here) more systems satisfy the uptake criterion. Fig. 11(c) shows a similar effect on the diagram of an increase in chemical potential, to $\Delta \mu^{*}=-8$. In both geometries, the altered criterion corresponds to more systems being allowed in the respective cavities. A different effect on the diagram occurs if the size of the nanotubes is changed, as shown in Fig. 11(d), under the thermodynamic conditions of Fig. 1(a). In the case of nanotubes with radius $8 \AA$ more atoms enter interstitial channels because of the larger channel space, while fewer atoms go inside the tubes because the adhesive energy decreases. The trends seen in Fig. 1(a)-(d) are qualitatively consistent with the behavior of $V_{\text {min }}^{*}$ presented in Figures 3 and 4 .

In the Henry's law regime of low coverage there is a convenient way to characterize the variation of uptake with geometry. We compute the ratio of particle occupations in the nanotubes and IC's at the same $P$ and $T$ :

$$
\Gamma\left(\epsilon_{g g}, \sigma_{g g}\right)=\frac{\nu_{N T}}{\nu_{I C}} \frac{\int_{N T} d \mathbf{r} \exp (-\beta V)}{\int_{I C} d \mathbf{r} \exp (-\beta V)}
$$


where $\nu_{N T(I C)}$ are the number of nanotubes (IC's) in the bundle and the integrations are over one region (assumed infinitely long). For an infinite array of nanotubes, $\nu_{N T} / \nu_{I C}=1 / 2$. The finiteness of the bundle changes the ratio; however, there is no qualitative effect on our conclusions unless the bundle is very small. This ratio depends on the two gas parameters, $\epsilon_{g g}$ and $\sigma_{g g}$. In order to simplify the presentation, we fit the general trend of systems in Table \ to an empirical equation:

$$
\epsilon_{g g}^{f i t} \simeq a \sigma_{g g}+b
$$

with values $a=147 \mathrm{~K} / \AA$ and $b=376 \mathrm{~K}$. We then consider a function of one variable

$$
\Gamma\left(\sigma_{g g}\right) \equiv \Gamma\left(\epsilon_{g g}^{f i t}, \sigma_{g g}\right)
$$

This ratio function is presented in Figures 7 and 8. In Fig. 7 we consider a common value of $T^{*}=1$, while in Fig.8, we consider a fixed $T=77 \mathrm{~K}$. The data in Fig. 7 shows, as expected, that large (small) molecules adsorb preferentially in the nanotubes (IC's). Fig. 8 differs for small $\sigma_{g g}$ because at $77 \mathrm{~K}$ the entropic advantage of the tubes is manifested as a larger uptake there than is seen in Fig. 7 at the much lower T given by Eq. (16).

\section{CONCLUSIONS}

Model calculations were used to investigate adsorption in nanotube bundles. Simplifying assumptions were made, such as the pairwise summation of gas-surface interactions, the use of combining rules to determine energy and size parameters, and the continuum, rigid model of the carbon atoms of the tube. We studied mainly the regime of low coverage, where interactions between adsorbed atoms are omitted; in the IC case, this assumption was not needed, as a quasi-one dimensional approximation permits exact treatment of LJ interactions at finite coverages. The conclusions drawn are expected to be qualitatively accurate in general situations, and so they provide useful insight for experiments. The key

result appears in Fig. 1, indicating which molecules go where under "typical" experimental conditions. More general behavior can be estimated from the reduced potential curves (Figures 3-5) we have presented. 
Williams and Eklund 31 have computed the $\mathrm{H}_{2}$ adsorption on the bounding surface of bundles containing a finite number of tubes. In some cases, this contribution can be a significant fraction of the total adsorption. Adsorption isotherms of classical gases on the external surface of the bundle is the subject of our current investigations to be reported in the future.

We discuss the relevant experiments very briefly. Teizer et al. [1] studied He uptake and found consistency with our calculations for one-dimensional motion and the computed binding energy within the interstitial channels. Kuznetsova et al. [9] studied uptake of Xe and their data are consistent with our calculations of the uptake within the nanotubes.

Interestingly, a recent experimental study of adsorption of methane in nanotube bundles [8] concluded that significant IC adsorption occurs. This conclusion was reached from the fact that nanotubes were capped and the measured binding energy of $\mathrm{CH}_{4}$ determined $(2570$ K) was $76 \%$ larger than that on graphite $(1460 \mathrm{~K})$ [47], which compared favorably with pre-

vious estimates of the $\mathrm{IC}$ binding energy of $\mathrm{H}_{2}, \mathrm{He}$ and $\mathrm{Ne}[12$ 14]. Our present calculations indicate, however, that the large size of $\mathrm{CH}_{4}$ prevent it from populating the narrow IC's significantly. In contrast, the external surface of the nanotube bundle is accessible and the binding energy in this case (Table $\mathbb{E}$ ) is $\sim 20 \%$ larger than the one for graphite. A more realistic potential exhibits corrugation, which we have neglected here; its effect is to increase the binding energy [13], but we have not undertaken that calculation as yet so no definite comparison is possible.

\section{ACKNOWLEDGMENTS}

We are grateful to Victor Bakaev, Moses Chan, Vincent Crespi, Peter Eklund, Karl Johnson, James Kurtz, Aldo Migone, Bill Steele and Keith Williams for useful discussions. This research was supported by the National Science Foundation, the Petroleum Research Fund of the American Chemical Society and the Army Research Office. One of the authors, S. C., would like to acknowledge the generous support of Fondazione Ing. Aldo Gini. 
[1] A. C. Dillon, K. M. Jones, T. A. Bekkedahl, C. H. Kiang, D. S. Bethune, and M. J. Heben, Nature 386, 377 (1997).

[2] A. Chambers, C. Park, R. T. K. Baker, and N. M. Rodriguez, J. Phys. Chem. B 102, 4253 (1998).

[3] S. Inoue, N. Ichikuni, T. Suzuki, T. Uematsu, and K. Kaneko, J. Phys. Chem. B 102, 4689 (1998).

[4] W. Teizer, R. B. Hallock, E. Dujardin, and T. W. Ebbesen, Phys. Rev. Lett. 82, 5305 (1999) and Erratum (in press).

[5] C. Nützenadel, A. Züttel, D. Chartouni, and L. Schlapbach, Electrochem. and Solid-State Lett. 2, 30 (1999).

[6] S. Orimo, G. Majer, T. Fukunaga, A. Züttel, L. Schlapbach, and H. Fujii, Appl. Phys. Lett. 75, 3093 (1999).

[7] E. B. Mackie, R. A. Wolfson, L. M. Arnold, K. Lafdi, and A. D. Migone, Langmuir 13, 7197 (1997).

[8] S. Weber, S. Talapatra, C. Journet, and A. D. Migone, "Adsorption of Methane on Single-Wall Nanotubes", Phys. Rev. (in press).

[9] A. Kuznetsova, J. T. Yates, Jr., J. Liu, and R. E. Smalley, "Physical Adsorption of Xenon in Open Single Walled Carbon Nanotubes-Observation of a Quasi 1-D Confined Xe phase", submitted to J. Chem. Phys.

[10] Y. Ye, C. C. Ahn, C. Witham, B. Fultz, J. Liu, A. G. Rinzler, D. Colbert, K. A. Smith, and R. E. Smalley, Appl. Phys. Lett. 74, 2307 (1999).

[11] P. Chen, X. Wu, J. Lin, K. L. Tan, Science, 285, 91 (1999).

[12] G. Stan and M. W. Cole, Surf. Sci. 395, 280 (1998) 
[13] G. Stan and M. W. Cole, J. Low Temp. Phys. 110, 539 (1998).

[14] G. Stan, M. Boninsegni, V. H. Crespi, and M. W. Cole, J. Low Temp. Phys. 113, 447 (1998).

[15] R. Radhakrishnan and K. E. Gubbins, Phys. Rev. Lett. 79, 2847 (1997).

[16] Q. Wang, S. R. Challa, D. S. Sholl, and J. K. Johnson, Phys. Rev. Lett. 82, 956 (1999).

[17] Y. F. Fin, T. Mays, and B. McEnaney, Langmuir 15, 8714 (1999).

[18] A. M. Vidales, V. H. Crespi, and M. W. Cole, Phys. Rev. B 58, R13426 (1998).

[19] F. Darkrim and D. Levesque, J. Chem. Phys. 109, 4981 (1998).

[20] C. Liu, Y. Y. Fan, M. Liu, H. T. Cong, H. M. Cheng, and M. S. Dresselhaus, Science 286, 1127 (1999).

[21] For a review of the up-to-date results in this area, see M. S. Dresselhaus, K. A. Williams, and P. C. Eklund, MRS Bull. 24, 45 (1999).

[22] E. Dujardin, T. W. Ebbesen, H. Hiura, and K. Tanigaki, Science 265, 1850 (1994).

[23] E. Cheng, M. W. Cole, W. F. Saam and J. Treiner, Phys. Rev. B 48, 18214 (1993).

[24] W. A. Steele, Chem. Rev. 93, 2355 (1993).

[25] W. A. Steele, Surf. Sci. 36, 317 (1973).

[26] G. Scoles, Intl. J. Quant. Chem. 24, 475 (1990).

[27] G. Ihm, M. W. Cole, F. Toigo and J. R. Klein, Phys. Rev. A 42, 5244 (1990).

[28] R. O. Watts and I. J. McGee, Liquid State Chemical Physics, (Wiley, New York, 1976).

[29] G. C. Maitland, M. Rigby, E. B. Smith, and W. A. Wakeham, Intermolecular forces: Their Origin and Determination (Clarendon Press, Oxford, 1987), tables A 3.1 and A 3.2, pp 565566.

[30] See L. W. Bruch, M. W. Cole, and E. Zaremba, Physical Adsorption: Forces and Phenomena, 
Appendix B (Oxford University Press, 1997). We note the distinction between the $\epsilon_{g g}$ value and the empirical well depth for the "true" pair potential. The latter is larger because the LJ value includes many-body effects.

[31] K. A. Williams and P. C. Eklund, "Monte Carlo Simulations of $\mathrm{H}_{2}$ Physisorption in FiniteDiameter Carbon Nanotube Ropes", submitted to Chem. Phys. Lett.

[32] H.-Y. Kim and M. W. Cole, Phys. Rev. B 35, 3990 (1987).

[33] As shown in Ref. [12], the equilibrium distance for atoms/molecules adsorbed inside a large nanotube $\left(R>1.2 \sigma_{g C}\right)$ is slightly greater than at a flat surface. In contrast, in small tubes and in IC's this distance may be significantly smaller than in the case of a flat surface.

[34] H.-Y. Kim and M. W. Cole, Surf. Sci. 194, 257 (1988).

[35] W. E. Carlos and M. W. Cole, Surf. Sci. 91, 339 (1980); L. W. Bruch, in Phase Transitions in Surface Films 2, H. Taub, G. Torzo, H. J. Lauter, and S. C. Fain, Jr., Eds., pp. 67-82 (Plenum, New York, 1991).

[36] A. Thess, R. Lee, P. Nikolaev, H. Dai, P. Petit, J. Robert, C. Xu, Y. H. Lee, S. G. Kim, A. G. Rinzler, D. T. Colbert, G. E. Scuseria, D. Tománek, J. E. Fischer, and R. E. Smalley, Science 273, 483 (1996).

[37] M. W. Cole, V. H. Crespi, G. Stan, J. M. Hartman, S. Moroni, and M. Boninsegni, "Anisotropic Condensation of Helium in Nanotube Bundles", submitted to Phys. Rev. Lett.

[38] For He, deviations from the classical prediction become important for these low densities only at cryogenic temperatures.

[39] A. Lotfi, J. Vrabec and J. Fischer, Mol. Phys. 76, 1319 (1992).

[40] M. Brack and R. K. Bhaduri, Semiclassical Physics (Addison-Wesley, 1997) pp. 75-78.

[41] M. J. Bojan and W. A. Steele, Carbon 36, 1417 (1998). 
[42] H. Takahashi, Mathematical Physics in One Dimension, E. H. Lieb, D. C. Mattis, Eds. (Academic Press, New York and London, 1966), pp. 25-27; F. Gürsey, Proc. Cambridge Phil. Soc. 46, $182(1950)$.

[43] For the external surface we have studied the film growth up to high coverage; we will report these results elsewhere.

[44] A. Cheng, M. L. Klein, and C. Caccamo, Phys. Rev. Lett. 71, 1200 (1993).

[45] B. W. Smith, M. Monthioux and D. E. Luzzi, Nature 396, 323 (1998).

[46] W. A. Steele, The Interactions of Gases with Solid Surfaces (Pergamon, Oxford, 1974).

[47] G. Vidali, G. Ihm, H.-Y. Kim, and M. W. Cole, Surf. Sci. Repts. 12, 133 (1991). 
TABLE I. The values of the LJ parameters, $\epsilon_{g g}$ and $\sigma_{g g}$, for the gas-gas interactions, the corresponding minimum $\left(V_{\min }\right)$ of the adsorption potential and ground state energies $\left(E_{0}\right)$ inside a nanotube $(N T)$, in the interstitial channel $(I C)$, on the external surface of the bundle (ext), and on a single graphite sheet $(G R)$ is given. LJ parameters were taken from Ref. [28], except for $\mathrm{CH}_{4}$, $\mathrm{CF}_{4}$ and $\mathrm{SF}_{6}$ [29] and $\mathrm{C}_{60}$ 44].

\begin{tabular}{ccccccccc}
\hline \hline $\mathrm{Gas}$ & $\epsilon_{g g}(\mathrm{~K})$ & $\sigma_{g g}(\AA)$ & $V_{\min }^{I C}(\mathrm{~K})$ & $E_{0}^{I C}(\mathrm{~K})$ & $V_{\min }^{N T}(\mathrm{~K})$ & $V_{\min }^{e x t}(\mathrm{~K})$ & $E_{0}^{e x t}(\mathrm{~K})$ & $V_{\min }^{G R}(\mathrm{~K})$ \\
\hline $\mathrm{He}$ & 10.2 & 2.56 & -546 & -386 & -297 & -367 & -270 & -218 \\
$\mathrm{Ne}$ & 35.6 & 2.75 & -1018 & -902 & -600 & -725 & -666 & -431 \\
$\mathrm{H}_{2}$ & 37.0 & 3.05 & -828 & -292 & -690 & -808 & -618 & -482 \\
$\mathrm{Ar}$ & 120 & 3.40 & 6 & 228 & -1426 & -1607 & -1550 & -965 \\
$\mathrm{CH}_{4}$ & 148 & 3.45 & 401 & 789 & -1614 & -1809 & -1714 & -1088 \\
$\mathrm{Kr}$ & 171 & 3.60 & 2048 & 2250 & -1836 & -2025 & -1981 & -1220 \\
$\mathrm{Xe}_{\mathrm{CF}}$ & 221 & 4.10 & 14786 & 15054 & -2523 & -2617 & -2580 & -1593 \\
$\mathrm{CF}_{4}$ & 157 & 4.58 & 36411 & 36854 & -2539 & -2475 & -2433 & -1520 \\
$\mathrm{SF}_{6}$ & 208 & 5.25 & 136492 & 137196 & -3726 & -3307 & -3272 & -2056 \\
$\mathrm{C}_{60}$ & 2300 & 9.2 & 52858932 & 52863770 & -49071 & -21952 & -21924 & -14505 \\
\hline \hline
\end{tabular}




\section{Figure Captions}

FIG. 1. (a) Diagram indicating regions of significant uptake (at thermodynamic conditions specified by $\Delta \mu^{*}=-10, T^{*}=1$ and $\rho^{*}=0.1$ ) as a function of the adsorbate Lennard-Jones parameters. Gases lying in the domain deoted "TUBE" are absorbed within the nanotubes. Those denoted "IC" are absorbed within the interstitial channels, while those denoted "BOTH" ("NEITHER") go to both places (neither place). Systems of particular interest are identified by dots $(\bullet)$, with parameters listed in Table —.

(b) Diagram analogous to (a), except that curves shown utilize an alternative $\rho^{*}$ value for the threshold condition, i.e. $\rho^{*}=0.05$.

(c) Same as in (a) for a different value of the chemical potential: $\Delta \mu^{*}=-8$.

(d) Same as in (a) in the case of a nanotube array with tubes of diameter $16 \AA$.

FIG. 2. Schematic picture of adsorption sites within and outside a nanotube bundle. For the external surface, the most attractive site, located at equal distance from two nanotubes, is shown here. Adsorbed atoms or molecules are represented by dots.

FIG. 3. Contour plot of the reduced well-depth $V_{m i n}^{*}$ of the adsorption potential inside a carbon nanotube. The attractive isopotential curves (-) correspond to $V_{\min }^{*}$ increments of 10 starting from -90 , while the repulsive curves $(--)$, from left to right, correspond to $V_{\min }^{*}=20,40$, and 80.

FIG. 4. Same as in Fig. 3, for the interstitial channel. Repulsive curves, from left to right, correspond to $V_{\min }^{*}=10,20,30$ and 40 .

FIG. 5. Same as in Fig. 3, for the external surface of the nanotube bundle.

FIG. 6. Expanded version of Fig. 1 1(a) showing the gas systems which absorb within a nanotube at $\rho^{*}=0.1, \Delta \mu^{*}=-10$ and $T^{*}=1$. 
FIG. 7. Ratio of the amount adsorbed inside a nanotube to that in an intersititial channel in the Henry's law (low coverage) regime. Here, $T^{*}=1$.

FIG. 8. Same as in Fig. [7, but for $T=77 \mathrm{~K}$. 


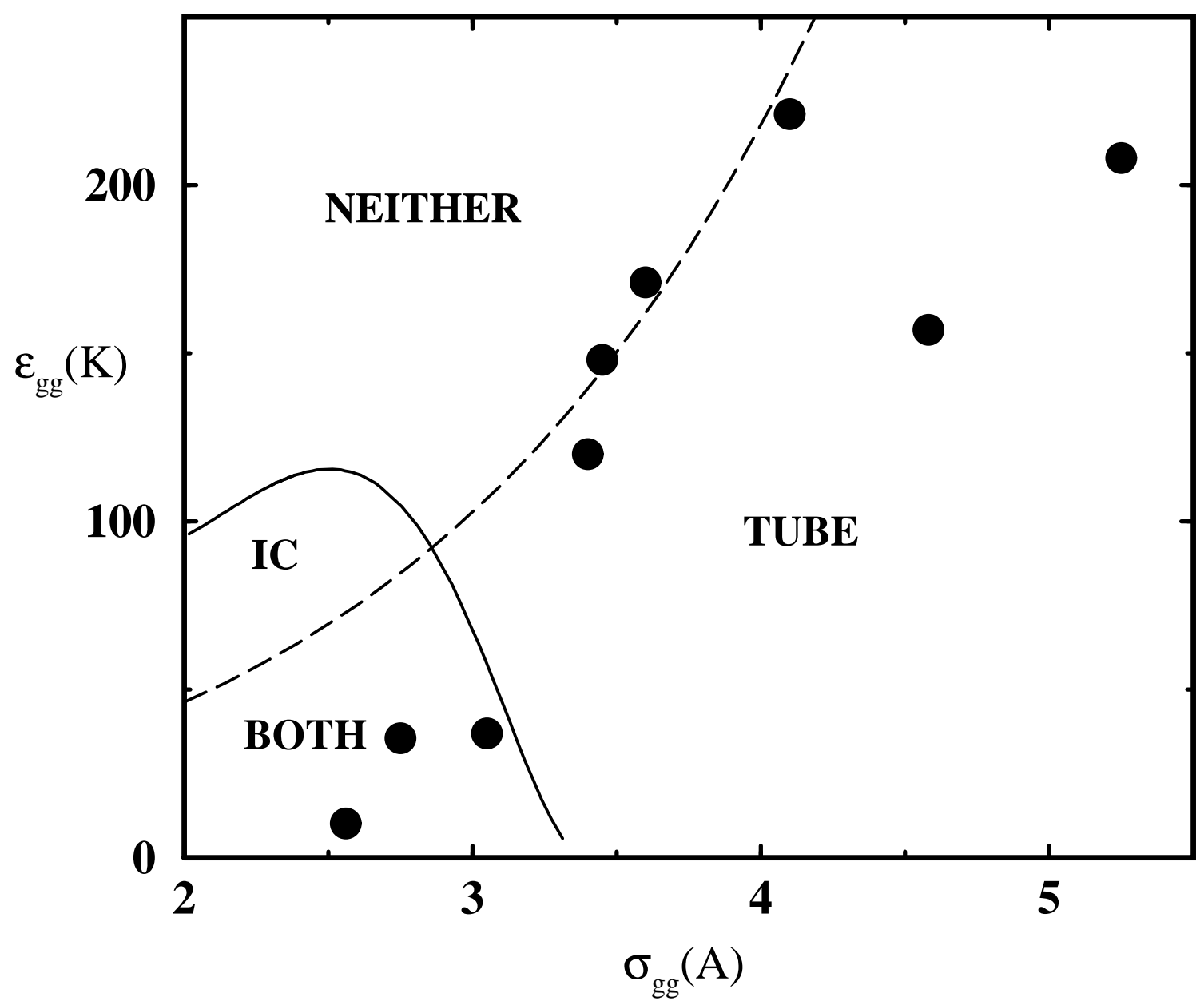

FIG. 11(a) 


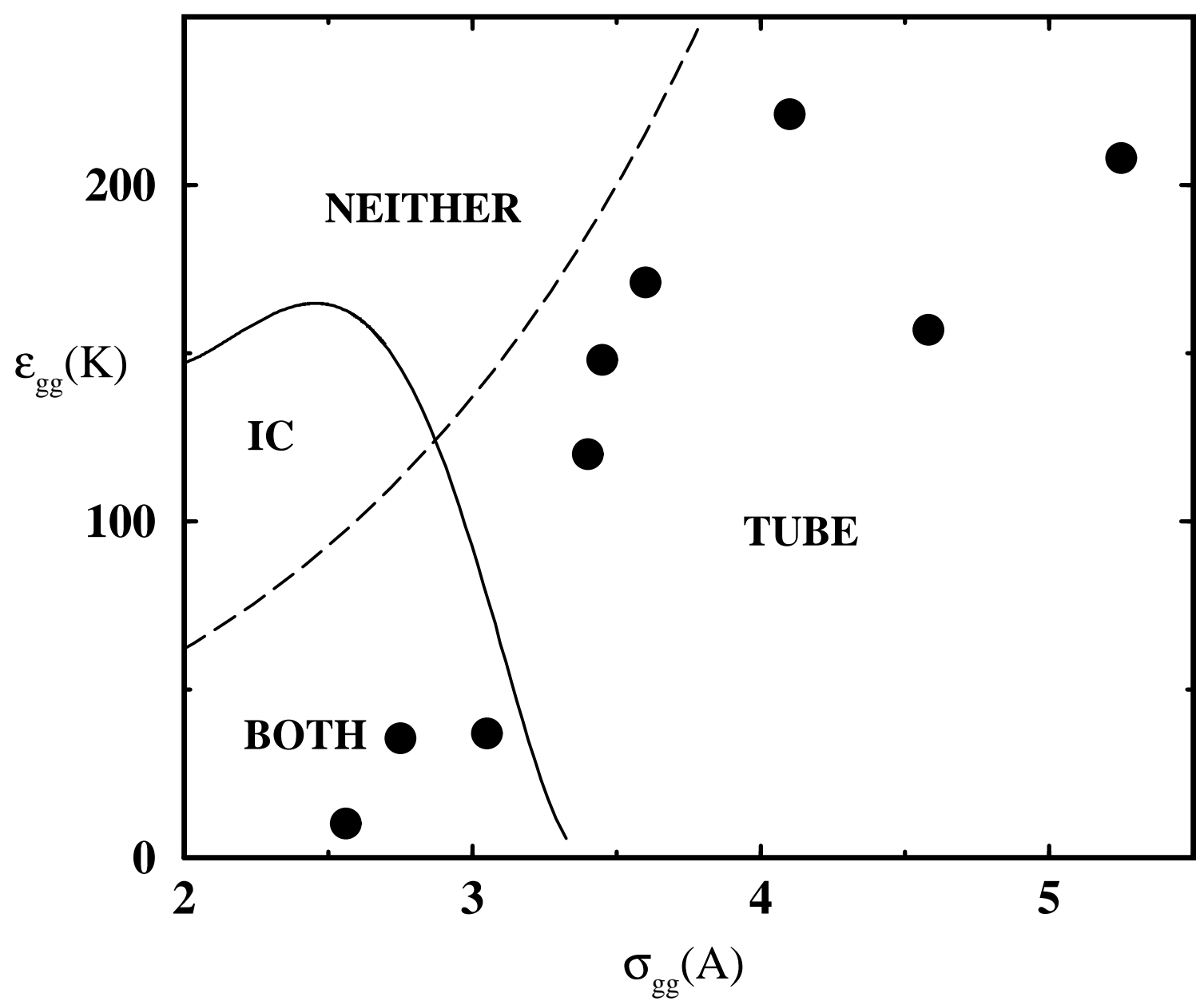

FIG. 11(b) 


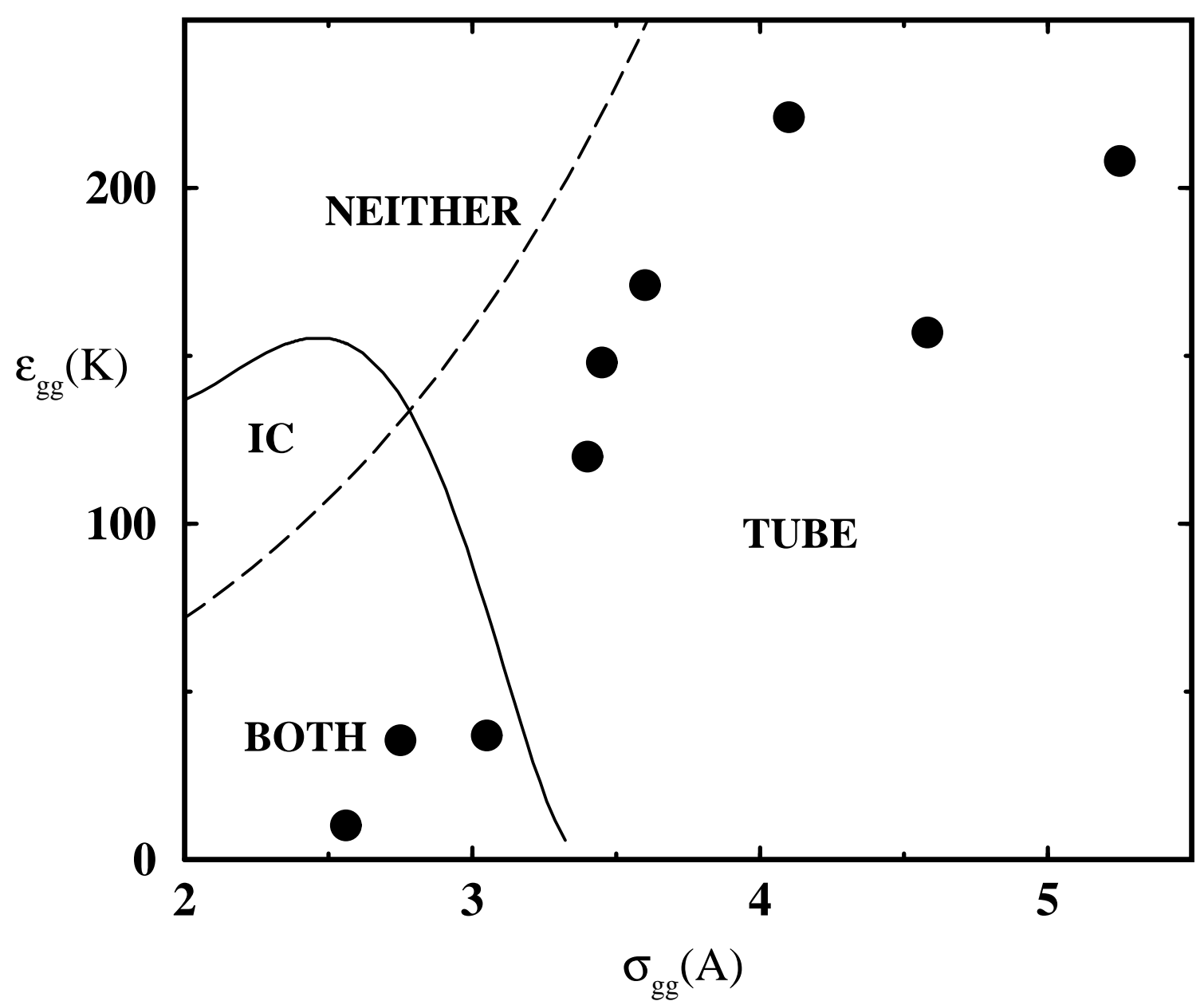

FIG. 1 $(\mathrm{c})$ 


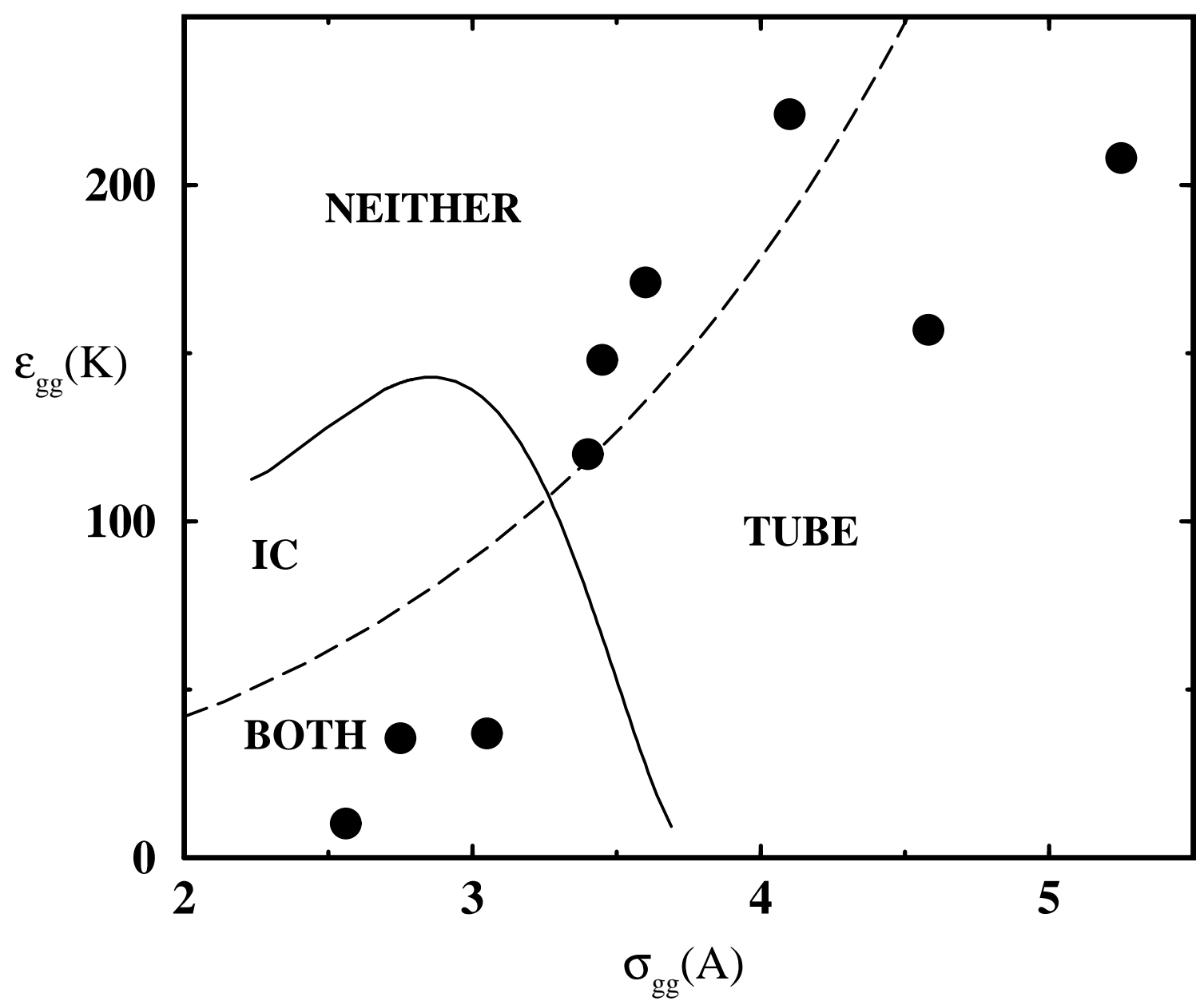

FIG. 11(d) 


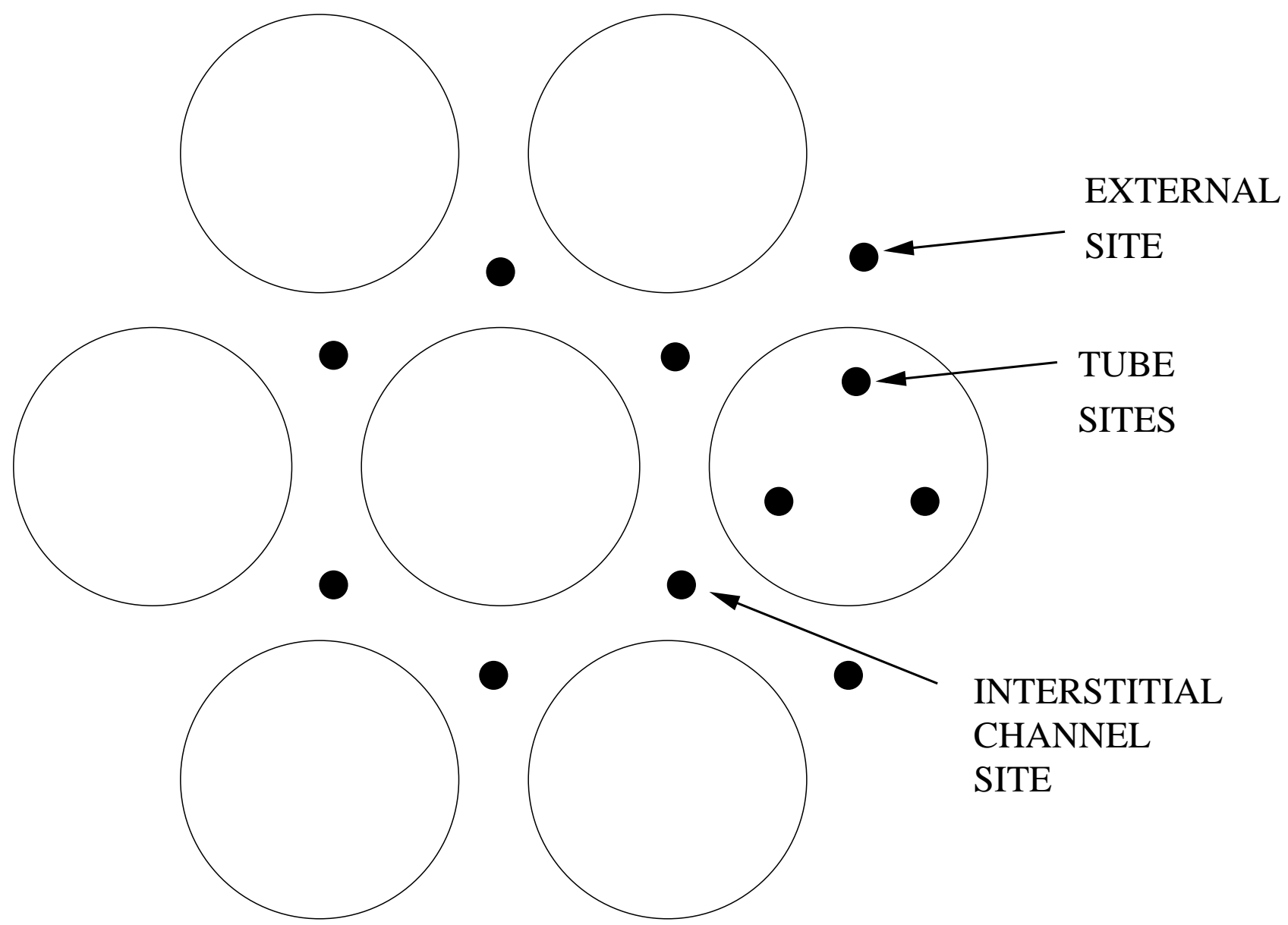

FIG. 2 


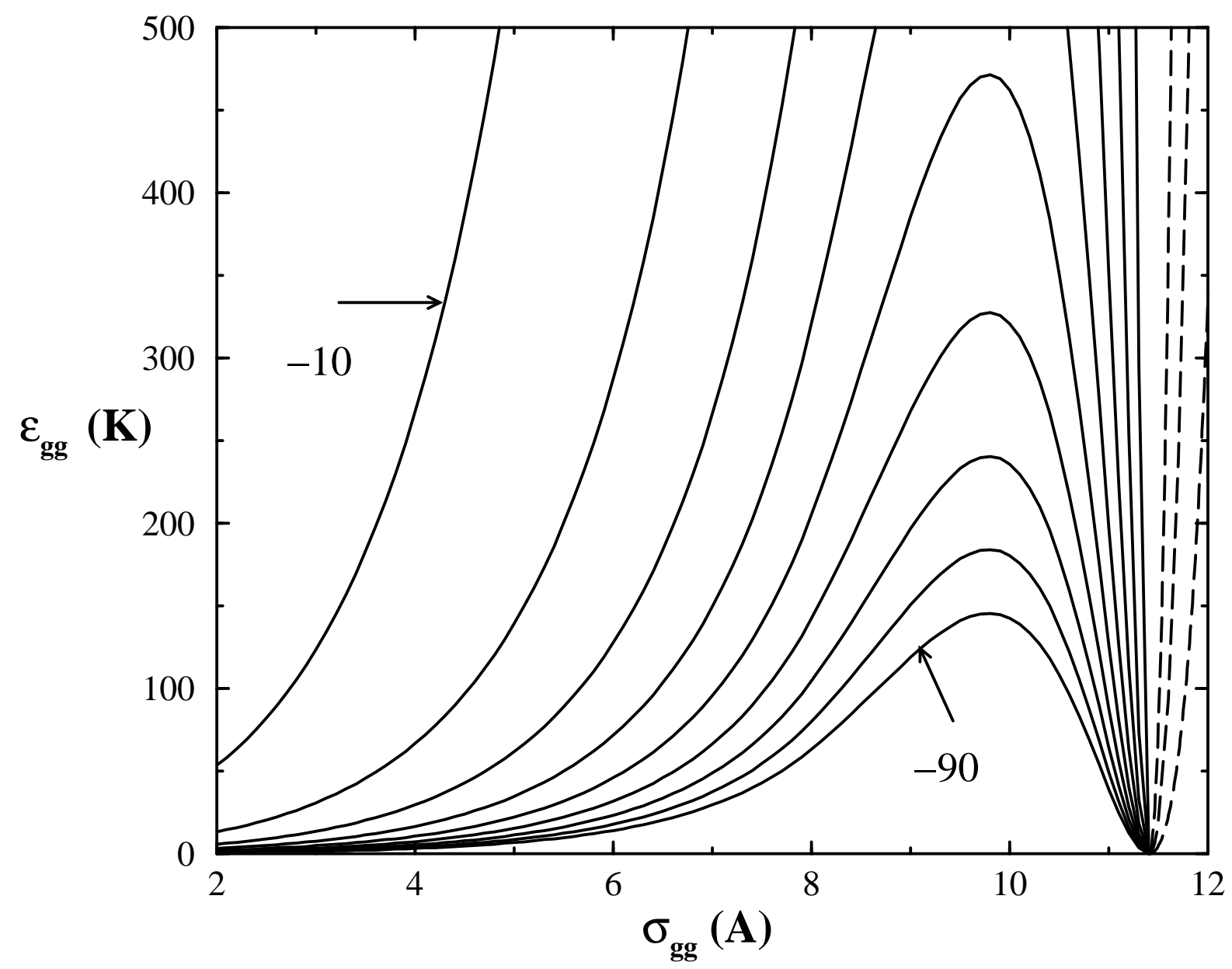

FIG. 3 


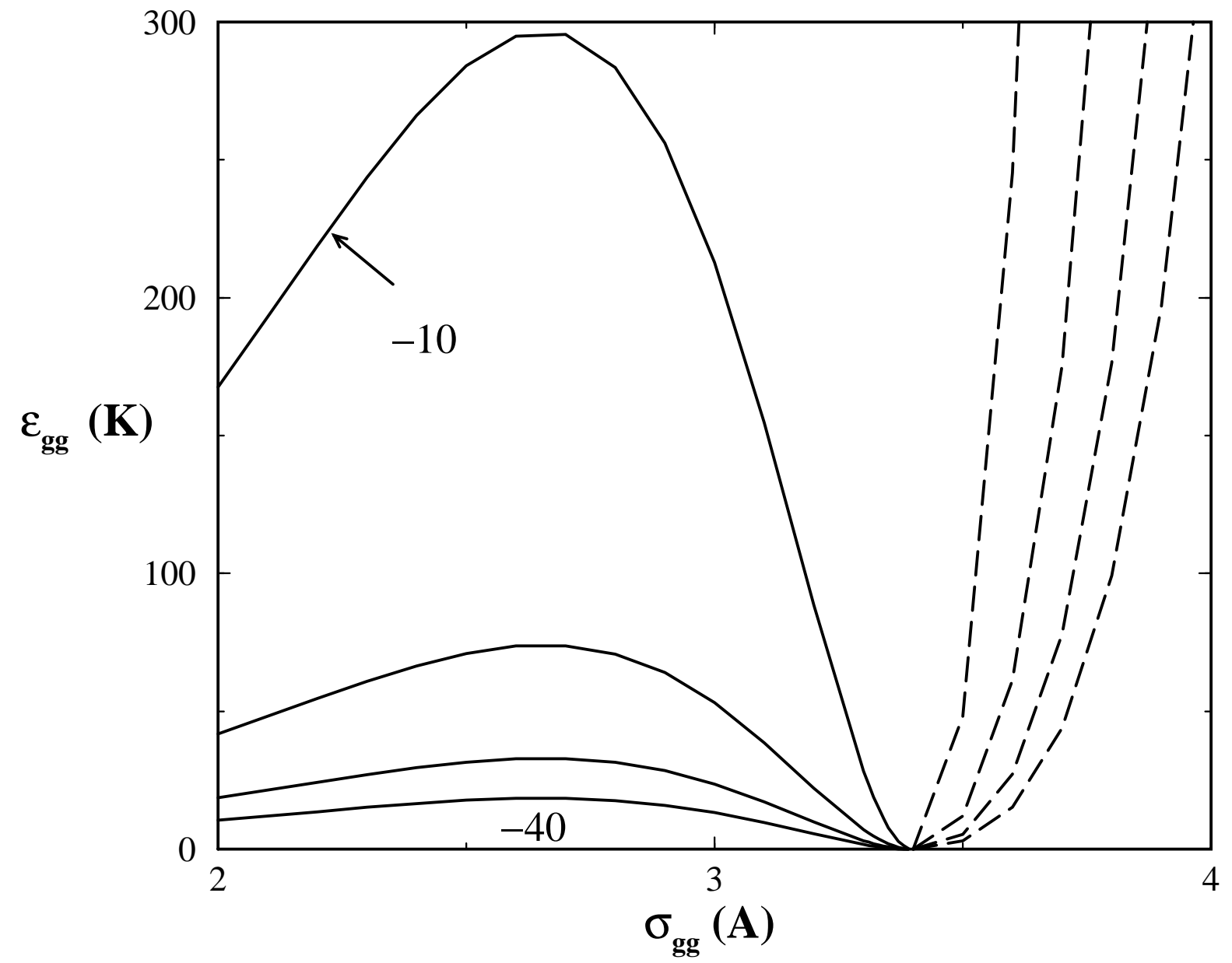

FIG. 1 


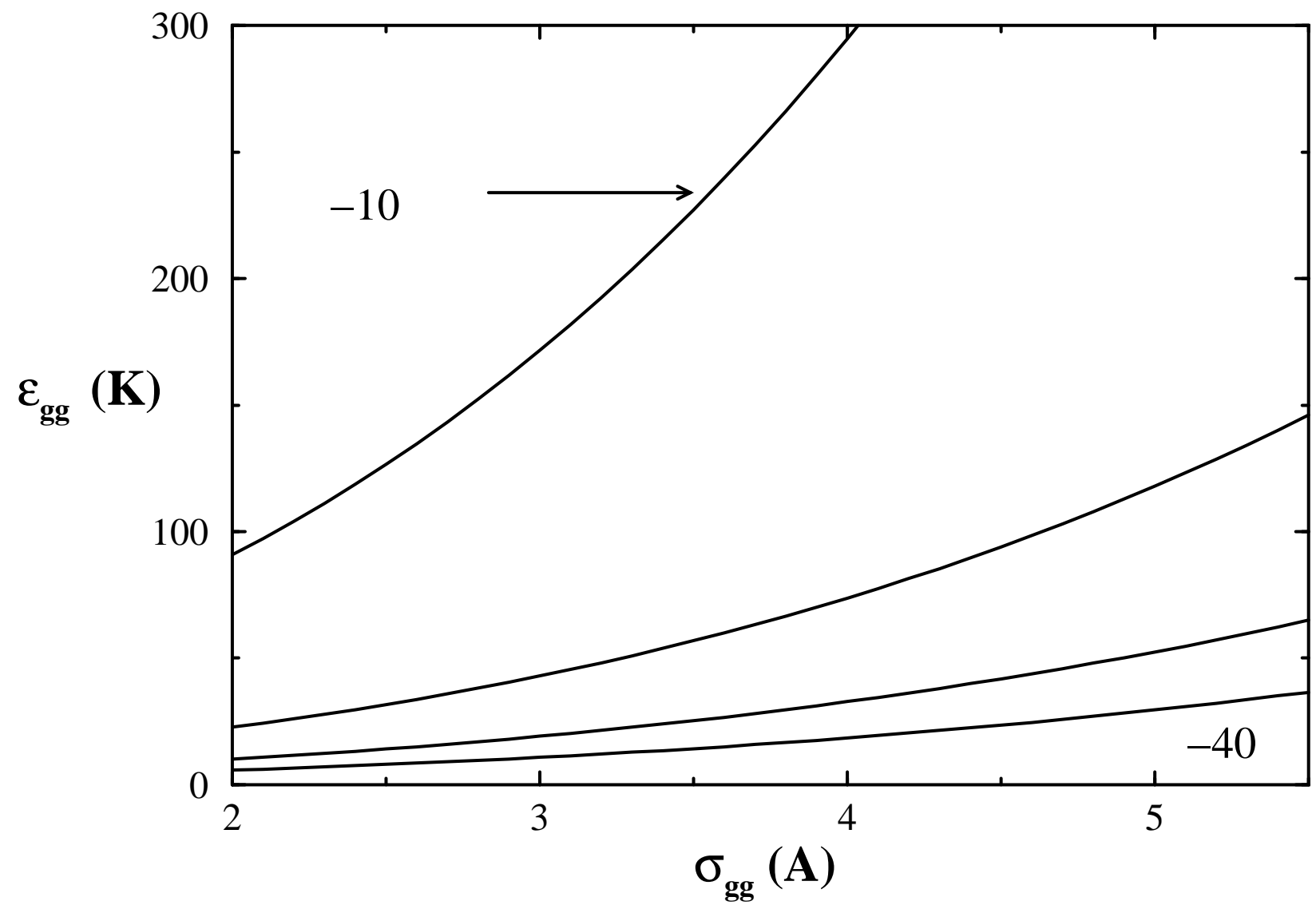

FIG. 5 


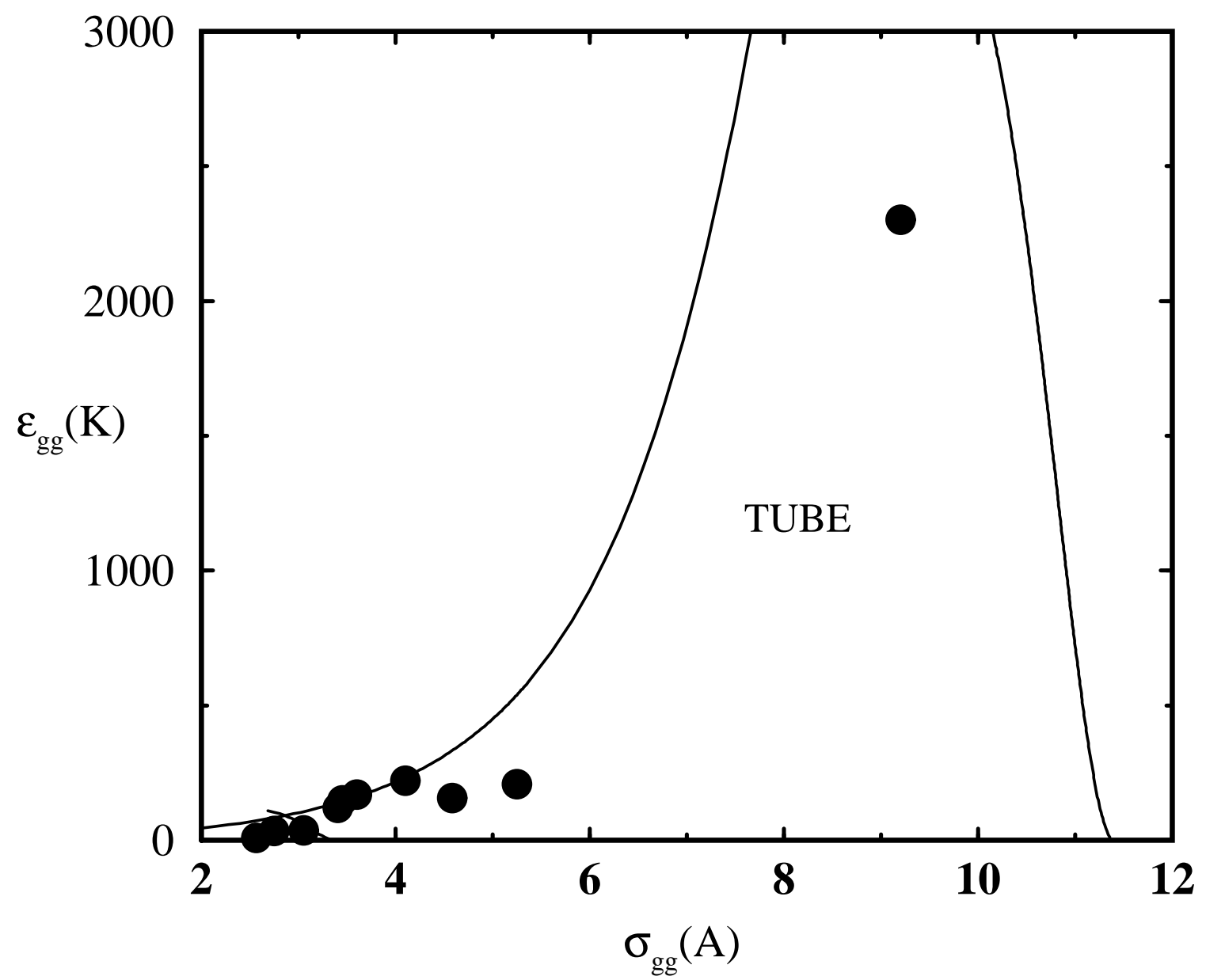

FIG. 6 


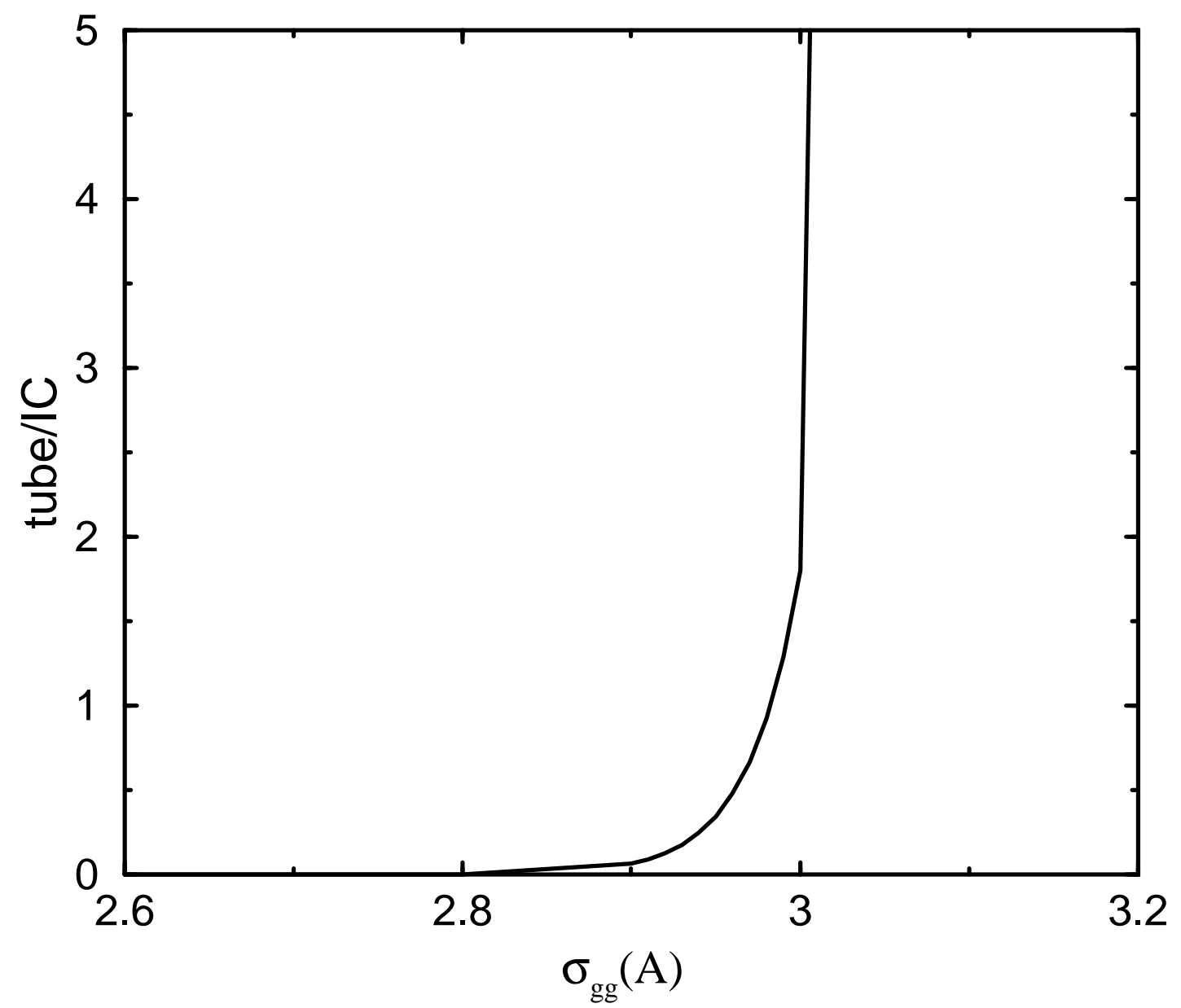

FIG. 7 


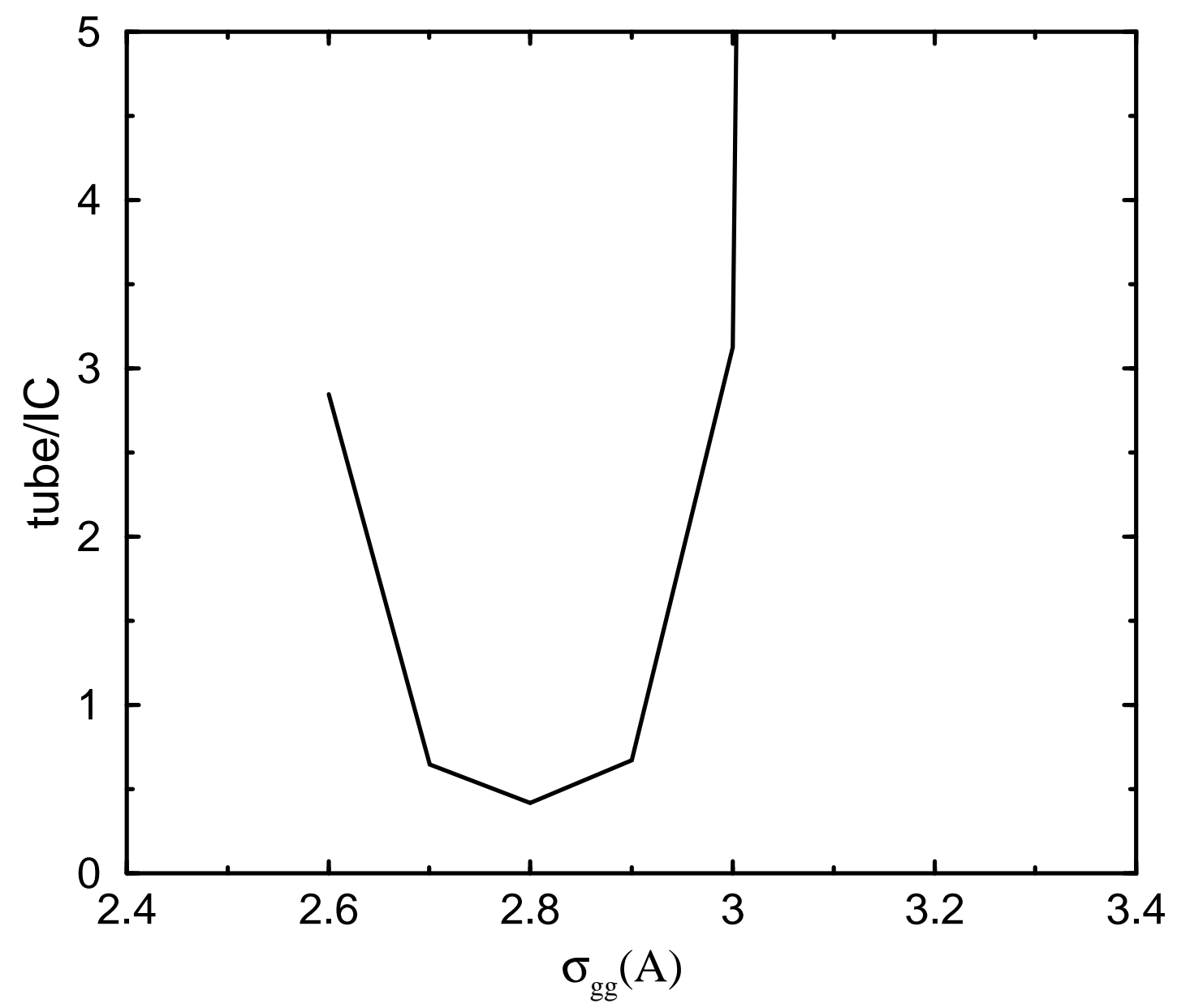

FIG. 8 